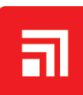 CHITKARA \\ Journal of Nuclear Physics, Material Sciences, Radiation and Applications \\ Journal homepage: https://jnp.chitkara.edu.in/
}

\section{Goodness of Generalized Seniority in Even-even Sn Isotopes}

\author{
Bhoomika Maheshwari* \\ Department of Physics, Banasthali Vidyapith, Banasthali-304022, India
}

*Email: bhoomika.physics@gmail.com

\section{ARTICLE INFORMATION}

Received: August 20, 2018

Revised: January 05, 2019

Accepted: February 08, 2019

Published online: February 26, 2019

\section{Keywords:}

Sn isotopes, Seniority, Generalized Seniority,

Isomers, B(E1), B(E2), B(E3), First excited

$2^{+}$and $3^{-}$states

DOI: $10.15415 /$ jnp.2019.62023

\begin{abstract}
Seniority has proved to be a unique and simple probe to address some of the complex issues underlying nuclear structure of nuclei close to magic numbers. An extension from the concept of seniority in single- $j$ shell to generalized seniority in multi-j shell has recently been provided by us. We have, consequently, established new selection rules for gamma decays and discovered the new seniority isomers decaying via odd electric multipole operators. We have successfully explained the $\mathrm{B}(\mathrm{EL} ; \mathrm{L}=1,2,3)$ behavior of various high spin isomers and other excited states. More specifically, we have been able to explain the long-standing puzzle of double hump in the B(E2) values for the first excited $2^{+}$states of eveneven $\mathrm{Z}=50(\mathrm{Sn})$ isotopes. In the present paper, we review these generalized seniority calculations with emphasis on even-even Sn isotopes. We first discuss the generalized seniority results for the E1 decaying 13- isomers and E2 decaying $10^{+}, 15^{-}$isomers, and then present the cases of first-excited $2^{+}$and $3^{-}$states. The generalized seniority proves out to be a reasonably good quantum number. The significance of configuration mixing is found to be true. The calculated results has been validated till high seniority $\mathrm{v}=4$ states and expected to be valid for higher seniority $\mathrm{v}=6, \ldots$ states also.
\end{abstract}

\section{Introduction}

The role of pairing interaction is important in understanding the structure of semi-magic nuclei. Seniority scheme, which is based on pairing interaction, was first introduced by Racah [1] to differentiate the states with same values of the orbital angular momentum $L$, the spin angular momentum $S$ and the total angular momentum $J$ for the LS-coupling approach in the atomic physics. This concept has similarly been embraced in nuclear structure physics $[2,3]$. This scheme accredits a simpler and truncated version than the complete shell model due to the incorporation of a limited number of allowed configurations.

Seniority is usually described as the number of unpaired nucleons. If all the particles are paired up to generate any state then the state will be known as seniority $v=0$ state. States having one broken pair of nucleons are said to be seniority $v=2$ states and so on. The concept has extensively been discussed in single-j orbital using the quasi-spin algebra $[4,5]$. The odd tensor electric/magnetic multipole operators hence conserve seniority by allowing transitions between the same seniority states only. A particle number independent variation of the $\mathrm{B}(\mathrm{E} 1)$, or $\mathrm{B}(\mathrm{E} 3) \ldots .$. , or $\mathrm{B}(\mathrm{M} 1)$ values is expected as a result. On the other hand, the even tensor electric/magnetic multipole operators may change seniority by allowing the transitions between the same seniority states (seniority conserving transitions), or the states having the seniority-difference by 2 (seniority changing transitions). This explains the parabolic trend of $\mathrm{B}(\mathrm{E} 2)$ values along with a dip, or peak at the middle of single-j shell. The seniority conserving E2 transitions support a dip while the seniority changing E2 transitions lead to the peak in the middle of $\mathrm{B}(\mathrm{E} 2)$ values. The good seniority states will follow the key features in their spectroscopic properties: i) a constant pairing energy gap, ii) the particle number independent energy variation and iii) a parabolic behavior of $\mathrm{B}(\mathrm{E} 2)$ values. For example, we can find almost vanishing $B(E 2)$ values at the middle for the transitions between the same seniority states. This further explains the origin of seniority isomers in single-j scheme along with their particle number independent energy variation [6-9].

$\mathrm{Sn}$ isotopes present a fertile ground to explore the seniority scheme because of the longest chain of isotopes. It is well known that the spacing between the ground state and the first excited $2^{+}$state is fairly constant throughout the full chain of $\mathrm{Sn}$ isotopes from the doubly magic ${ }^{100} \mathrm{Sn}(\mathrm{Z}=50$, $\mathrm{N}=50)$ to the next doubly magic ${ }^{132} \mathrm{Sn}(\mathrm{Z}=50, \mathrm{~N}=82)$. The key features highlighting the goodness of seniority for these states exist throughout the full chain having multi-j orbitals. A multi-j description of the seniority scheme is therefore 
needed. Arima and Ichimura [10] first generalized the seniority scheme to multi-j orbitals. Talmi provided a detailed and rigorous investigation of the generalized seniority scheme $[11,12]$. We have recently explored this scheme for multi-j degenerate orbitals having different parities $[13,14]$ and established a new set of "generalized seniority" selection rules $[15,16]$. Seniority isomers, which were qualitatively known in E2 decaying states only, have now been found in E1 decaying states along with a quantitative description. Our calculations could hence establish a new type of seniority isomers decaying by odd electric multipole tensors. These results also reproduce the $\mathrm{B}(\mathrm{EL} ; \mathrm{L}=1,2,3 \ldots$, irrespective of the nature of tensor) properties of various states in semimagic nuclei [15-19]. The inclusion of configuration mixing is found to be essential.

In this paper, we present a brief review on our understanding of the even-even $\mathrm{Sn}$ isotopes in terms of the generalized seniority scheme based on our earlier works. We first discuss the new kind of seniority isomers, particularly the E1 decaying 13 - isomers in $\mathrm{Sn}$ isotopes. The calculated results reproduce the experimental trend and highlight the role of configuration mixing. The generalized seniority remains to be $v=4$ for these isomers. We then present the well-known case of $10^{+}$isomers having seniority $v=2$ in pure $\mathrm{h}_{11 / 2}[20,21]$ by using our generalized seniority scheme. We find and validate these isomers as generalized seniority $v=2$ isomers by explaining the full $\mathrm{B}(\mathrm{E} 2)$ trend from the suggested configuration mixing. We then compare these results with the 15 isomers in $\mathrm{Sn}$ isotopes having generalized seniority $v=4$. The $\mathrm{B}(\mathrm{E} 1)$ trend in $13^{-}$isomers is found to be similar with that of $\mathrm{B}(\mathrm{E} 2)$ trends in $10^{+}$and $15^{-}$isomers due to the goodness of generalized seniority.

We further discuss the results for the very first excited $2^{+}$and $3^{-}$states of even-even $\mathrm{Sn}$ isotopes. We have explained the origin of inverted $\mathrm{B}(\mathrm{E} 2)$, or $\mathrm{B}(\mathrm{E} 3)$ parabolas for both the states in terms of seniority changing transitions (in different seniority states having the seniority-difference as 2). The dip in the middle of $\mathrm{B}(\mathrm{E} 2)$ trend for these $2^{+}$states was found as a puzzle in contrast to the expected peak from the pure seniority scheme. It has now been attributed to the change in configurations, before and after the middle, by using the generalized seniority scheme. However, the states are of generalized seniority $v=2$ throughout the chain. In this way, we explained this long- standing anomaly in a simple manner. On the other hand, the $\mathrm{B}(\mathrm{E} 3)$ trend for these first excited $3-$ states has been explained via the $d-h$ orbitals as generalized seniority $v=2$ states.

Therefore, "generalized seniority" is found to behave as a good quantum number for the various states of even-even $\mathrm{Sn}$ isotopes. The generalized seniority is found to be valid till large-j value of $11 / 2$, whereas the seniority scheme is conserved till $j=7 / 2$. Predictions have also been made for the gaps in the measurement.
In section 2, we present the theoretical details of generalized seniority and their reduction formulae for multi-j orbitals. Section 3 , discusses the calculations and results along with the details of configuration mixing and the chosen subspace for various states in even-even $\mathrm{Sn}$ isotopes. Section 4 concludes the paper and explains how the generalized seniority governs the electromagnetic properties in Sn isotopes.

\section{Theoretical formalism: Generalized seniority scheme}

Arima and Ichimura [10] firstly introduced the idea of generalized seniority in multi-j degenerate orbitals. Talmi then extended the idea to many non-degenerate orbitals and applied it to study the semi-magic nuclei $[11,12]$. They generalized the seniority scheme by introducing a new pair creation operator for multi-j situation, which can be termed as the generalized pair creation operator, by summing over the pair creation operators of single-j $[4,6]$ and defined as

$$
S^{+}=\sum_{j} S_{j}^{+}
$$

The SU(2) Lie algebra holds for the generalized pair creation and generalized pair annihilation operators, where $S^{+}, S, S^{0}$ are the new generators. Thus,

$$
\left[S^{+}, S^{-}\right]=\sum_{j m} a_{j m}^{+} a_{j m}-\frac{1}{2} \sum_{j}(2 j+1)=\sum_{j} n_{j}-\Omega=2 S^{0}
$$

$\therefore\left[S^{0}, S^{+}\right]=S^{+},\left[S^{0}, S^{-}\right]=-S^{-}$

One can also obtain the energy values of the pairing interaction $2 S^{+} S^{-}=2\left(S^{2}-S^{0}\left(S^{0}-1\right)\right)$, in multi-j as

$2 s(s+1)-\frac{1}{2}(\Omega-n)(\Omega+2-n)=\frac{n-v}{2}(2 \Omega+2-n-v)(3)$ where the quasi-spin $s=\frac{1}{2}(\Omega-v)$ is related to the pair degeneracy of multi-j $\Omega=\frac{1}{2} \sum_{j}(2 j+1)$ and $n=\sum_{j} n_{j}$ presents the total number of particles.

The single-j scheme algebra concludes that the odd tensor electric/magnetic multipole operators become quasispin scalars and thus conserve the seniority by allowing the transitions between the same seniority states. This leads to the particle number independent variation of $\mathrm{B}(\mathrm{EL})$, or $\mathrm{B}(\mathrm{ML})$ values. The even tensor electric/magnetic multipole operators become the zero component of a quasi-spin vector and may allow the change in seniority. This further explains the parabolic variation of $\mathrm{B}(\mathrm{E} 2)$ values with a dip in the middle of the shell for the E2 transitions between the same seniority states. This explains the origin of seniority isomers in E2 decaying transitions in single-j scheme. Also, the 
origin of an inverted B(E2) parabola has been expected for the $\mathrm{E} 2$ transitions between the states differ in seniority by 2 .

Additionally, in the multi-j quasi spin scheme with several degenerate-j orbitals for $j \neq j$, the $S^{+}=\sum_{j} S_{j}^{+}$ (generalized pair creation operator) and Hermitian operator $T$ of rank $k$, can be related via the following commutation relation:

$$
\begin{aligned}
& {\left[S^{+}, T_{\kappa}^{(k)}\right]=\frac{1}{\sqrt{2 k+1}} \sum_{j<j^{\prime}}\left[1+(-1)^{k}\right]\left(j\left\|T_{\kappa}^{(k)}\right\| j^{\prime}\right)} \\
& \left(a^{j} \times a^{j^{\prime}}\right)_{\kappa}^{(k)}
\end{aligned}
$$

In result, the Hermitian operator $T$ having rank $k$ becomes a quasi-spin scalar for odd- $k$ values since the commutation relation results in zero. While it behaves as the zero component of a quasi-spin vector for even- $k$ values since the commutation relation will always result in non-zero value for this case [6]. A simple generalization was, therefore, expected from the single-j scheme. Still, a quantitative description was not available so far.

We have further used the pair creation operator for the case of multi-j orbitals having different parities $[13,14]$ as $S_{1}^{+}=\sum_{j}(-1)^{l_{j}} S_{j}^{+}$, where $l_{j}$ presents the value of orbital angular momentum quantum number for the given $j$-orbital. Due to this extra phase factor of parities, the $(-1)^{k}$ in equation (4) is now modified as $(-1)^{4+l+L}$, where $L$ is multipolarity (nature) of the transition in case of electromagnetic transitions. $l$ and $l$ 'represent the parities of initial and final states, respectively, for the given transition. Therefore,

(i) when $(-1)^{l+l^{l}+L}=-1$, the electro-magnetic operator of "even or odd tensor", behaves as a quasi-spin scalar. Interestingly, this will be true for the magnetic multipole transitions only, irrespective of the involved parities $l, l$ ' and nature of tensor $L$.

(ii) when $(-1)^{1+l^{*} L}=+1$, the electro-magnetic operator of "even or odd tensor", becomes a quasi-spin vector with zero component. This case is only true for the electric multipole transitions, irrespective of the involved parities $l, l$, and nature of tensor $L$.

(iii) As a consequence, we arrive at a new set of "generalized seniority" selection rules and a new situation to find seniority isomerism.

We can then simply obtain the reduced electric transition probabilities in multi-j scheme by defining $\tilde{j}=j \otimes j^{\prime}$...., using the well-known quasi-spin algebra, with the generalized pair degeneracy $\Omega=\frac{1}{2}(2 \tilde{j}+1)=\frac{1}{2} \sum_{j}(2 j+1)$, as

$$
B(E L)=\frac{1}{2 J_{i}+1} \|\left.\left\langle\tilde{j}^{n} v l J_{f}\left\|\sum_{i} r_{i}^{L} Y^{(L)}\left(\theta_{i}, \phi_{i}\right)\right\| \tilde{j}^{n} v^{\prime} l^{\prime} J_{i}\right\rangle\right|^{2}
$$

where the reduced matrix elements in equation (7) are described in the $\tilde{j}^{n}$ configuration. This may be related to the reduced matrix elements in $\tilde{j}^{v}$ configuration, as the following generalized seniority reduction formulae:

$$
\begin{aligned}
& \left\langle\tilde{j}^{n} v l J_{f}\left\|\sum_{i} r_{i}^{L} Y^{(L)}\left(\theta_{i}, \phi_{i}\right)\right\| \tilde{j}^{n} v^{\prime} l^{\prime} J_{i}\right\rangle=\left[\frac{(\Omega-n)}{(\Omega-v)}\right] \\
& \left\langle\tilde{j}^{v} v l J_{f}\left\|\sum_{i} r_{i}^{L} Y^{(L)}\left(\theta_{i}, \phi_{i}\right)\right\| \tilde{j}^{v} v^{\prime} l^{\prime} J_{i}\right\rangle, \text { for } \Delta v=0 \\
& \left\langle\tilde{j}^{n} v l J_{f}\left\|\sum_{i} r_{i}^{L} Y^{(L)}\left(\theta_{i}, \phi_{i}\right)\right\| \tilde{j}^{n}, v \mp 2, l^{\prime} J_{i}\right\rangle \\
& =\left[\sqrt{\frac{(n-v+2)(2 \Omega+2-n-v)}{2(2 \Omega+2-2 v)}}\right] \\
& \left\langle\tilde{j}^{v} v l J_{f}\left\|\sum_{i} r_{i}^{L} Y^{(L)}\left(\theta_{i}, \phi_{i}\right)\right\| \tilde{j}^{v}, v \mp 2,,^{\prime} l^{\prime} J_{i}\right\rangle, \text { for } \Delta v=2
\end{aligned}
$$

Hence, the resulting $\mathrm{B}(\mathrm{EL})$ trend depends on the generalized pair degeneracy $(\Omega)$, the total particle number in multi- $\mathrm{j}(n)$ and the generalized seniority $(v)$ according to the coefficients in the square brackets of equations (8) and (9), for seniority conserving $(\Delta v=0)$, and seniority changing $(\Delta v=2)$ transitions, respectively. To sum up, the generalized seniority scheme dictates the following electromagnetic properties:

(i) The reduced magnetic multipole transition probabilities, i.e. $\mathrm{B}(\mathrm{M} 1 / \mathrm{M} 2 / \mathrm{M} 3 \ldots)$ values will always result in the particle number independent variation. So, the magnetic moments and the related g-factors will also exhibit a particle number independent variation.

(ii) The reduced electric multipole transition probabilities, i.e. $\mathrm{B}(\mathrm{E} 1 / \mathrm{E} 2 / \mathrm{E} 3 \ldots)$ values exhibit a parabolic variation along with a dip at the middle for seniority conserving $\Delta v=O$ transitions, or with a peak at the middle for seniority changing $\Delta v=2$ transitions.

(iii) This leads to a new situation for observing the seniority isomers in $\mathrm{E} 1 / \mathrm{E} 3 \ldots$ decaying states in contrast to the general belief of $\mathrm{E} 2$ decaying seniority isomers only.

The states, which follow these generalized seniority electromagnetic selection rules, will also exhibit a particle 
number independent energy variation throughout the given multi-j configuration space.

\section{Results and discussion}

\subsection{A new kind of isomers: E1 decaying $13^{-}$ isomers}

Seniority isomers are, so far, known in E2 transitions [6-9], as no favorable conditions exist for the seniority isomers in E1/E3.... transitions within the single-j scheme. Recent experimental studies $[22,23]$ have reported the $15^{-}$and $13^{-}$isomers (seniority $v=4$ states) in even-even $\mathrm{Sn}$ isotopes. A parabolic B(E2) behavior for the 15 - isomers has been noted. Besides, a similar parabolic trend for the $\mathrm{B}(\mathrm{E} 1)$ values of 13 isomers has also been found surprisingly [23]. This issue involving different parity orbitals from the active valence space can now be taken into account due to the new generalized seniority selection rules. We first present the case of 13 isomers by freezing the $\mathrm{g}_{7 / 2}$ and $\mathrm{d}_{5 / 2}$ orbitals as fully filled till ${ }^{114} \mathrm{Sn}$ (an assumed core).

The $\mathrm{B}(\mathrm{E} 1)$ trend, for the $13^{-}$isomers, has been calculated by using the configuration mixing of $\tilde{j}=h_{11 / 2} \otimes d_{3 / 2} \otimes s_{1 / 2}, \Omega=9 ;$ assuming the generalized seniority $v=4$ in $\Delta v=0$ transitions. The top panel of Fig. 1 exhibits the calculated and experimental B(E1) trends for the $13^{-}$isomers. The generalized seniority calculated trend is line with the experimental data [23]. The configuration mixing $\tilde{j}=h_{11 / 2} \otimes d_{3 / 2} \otimes s_{1 / 2}, \Omega=9$ is required to obtain the full $\mathrm{B}(\mathrm{E} 1)$ trend for the $13^{-}$isomers. This is in contrast to the configuration mixing $h_{11 / 2}^{3} \otimes d_{3 / 2}^{1}$ as reported by Iskra et al. [23]. The other omega values $(\Omega=7$, or 8$)$ yield far from the measurements [15]. The generalized seniority of 13 isomers turns out to be constant at $v=4$. The measured relative energy spacing between the $13^{-}$and $10^{+}$isomers turns out to be fairly constant on going from ${ }^{120} \mathrm{Sn}$ to ${ }^{128} \mathrm{Sn}$. This is an experimental evidence for the goodness of generalized seniority for these $13^{-}$isomers. Hence, we could establish the E1 (odd tensor) decaying seniority isomers for the first time. Predictions for the 13 isomers at ${ }^{118,128} \mathrm{Sn}$ can be made via these results, where the $\mathrm{B}(\mathrm{E} 1)$ values are expected to be in the order of parabolic behavior.

\subsection{Importance of configuration mixing in E2 decaying $10^{+}$and $15^{-}$isomers}

The $10^{+}$isomers have generally been designated as pure seniority $v=2, \mathrm{~h}_{11 / 2}$ states $[6,20,21]$. Interestingly, the $\mathrm{h}_{11 / 2}$ orbital can only occupy a total of 12 particles, while the experimental $\mathrm{B}(\mathrm{E} 2)$ trend exists from ${ }^{116} \mathrm{Sn}$ to ${ }^{130} \mathrm{Sn}$. Therefore, a generalized seniority treatment by incorporating the multi-j situation is needed to explain the full $B(E 2)$ trend. We have calculated $\mathrm{B}(\mathrm{E} 2)$ values of these isomers as $v=2$ arising from $\tilde{j}=h_{11 / 2} \otimes d_{3 / 2} \otimes s_{1 / 2}, \Omega=9$ in $\Delta v=0$ transitions. The experimental and generalized seniority calculated B(E2) values for these isomers have been shown in Fig. 1, see middle panel. These calculations explain the experimental data $[20,21,24]$ quite well, except for a deviation at ${ }^{128,130} \mathrm{Sn}$. This may be due to the dominated as well as constant occupancy of $\mathrm{h}_{11 / 2}$ at $\mathrm{N}=78$ and 80 . The suggested configuration mixing (by generalized seniority) is found to be important in explaining the $\mathrm{B}(\mathrm{E} 2)$ values of $10^{+}$ isomers quantitatively in contrast to the general belief (in terms of pure seniority). A detailed comparison between the pure seniority scheme and the generalized seniority scheme has already been presented for these isomers [15].

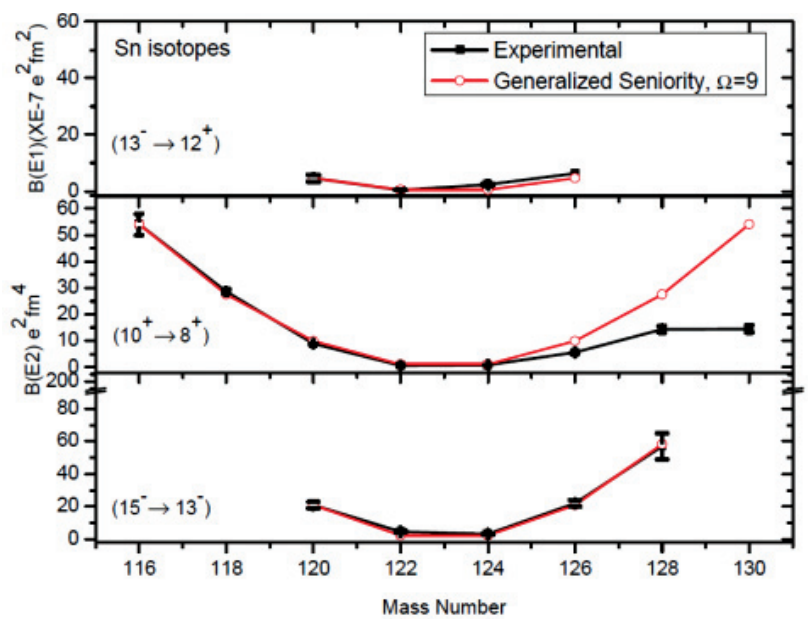

Figure 1: Experimental and Generalized seniority calculated $\mathrm{B}(\mathrm{E} 1)$, or $\mathrm{B}(\mathrm{E} 2)$ variation for the seniority conserving transitions in even-even $\mathrm{Sn}$ isotopes: top panel for E1 decaying $13^{-}$isomers, middle panel for E2 decaying $10^{+}$isomers and the bottom panel for E2 decaying $15^{-}$isomers, respectively.

Similarly, the calculations for B(E2) values of the 15 isomers have been done as generalized seniority $v=4$ states by using $\tilde{j}=h_{11 / 2} \otimes d_{3 / 2} \otimes s_{1 / 2}, \Omega=9$ in $\Delta v=4$ transitions. The calculated trend explains the experimental data quite well, see Fig. 1 (bottom panel). It is noted that both the $10^{+}$and $15^{-}$isomers show similar parabolic B(E2) trends, however, the involved seniorities are different. We have fixed proportionality constants by fitting the first available measured value of $\mathrm{B}(\mathrm{E} 1)$, or $\mathrm{B}(\mathrm{E} 2)$ trends (on the extreme left of Fig. 1 in all the panels respectively) to obtain the numeric numbers. These constants also provide a cue on the radial part of the interaction in multi-j scheme. We predict the $\mathrm{B}(\mathrm{E} 2)$ value of $15^{-}$isomers at ${ }^{118} \mathrm{Sn}$, in the order of $\mathrm{B}(\mathrm{E} 2)$ value of $15^{-}$isomers at ${ }^{128} \mathrm{Sn}$ through these investigations. All the $10^{+}, 13^{-}$and $15^{-}$isomers can be explained via the generalized seniority scheme. That's why the similar 
parabolic behavior of $\mathrm{B}(\mathrm{E} 1)$ and $\mathrm{B}(\mathrm{E} 2)$ values is expected and obtained.

\subsection{Twin B(E2) parabolas for the first excited $2^{+}$states}

It is well known that the first excited $2^{+}$states exhibit a particle number independent energy variation from ${ }^{100} \mathrm{Sn}$ to ${ }^{130} \mathrm{Sn}$. This further suggests that seniority may be good leading in their $\mathrm{B}(\mathrm{E} 2)$ behavior. An inverted parabolic $\mathrm{B}\left(\mathrm{E} 2 ; 0^{+} \rightarrow 2^{+}\right)$behavior was hence expected. Many theories [25-30] as well as experiments [31-44] reported and discussed the associated peak in the middle of the B(E2) curve. In contrast to this, a dip at ${ }^{116} \mathrm{Sn}$ (middle) has recently been noticed which was attributed due to reduced collectivity [31]. Morales et al. [25] have then investigated this minimum and explained via the different rates of filling of the orbitals. Different theoretical probes, such as core polarization, multiple sets of single-particle energies etc. have been used to understand this deviation at the middle [29, 30]. Many doubts are still lingering on. We have therefore planned to study these $\mathrm{B}(\mathrm{E} 2)$ values using generalized seniority scheme. We first re-evaluated the measured $\mathrm{B}(\mathrm{E} 2)$ data in view of the different measurements available [45]; a list of evaluated values has been given in the previous paper [16]. We compare the evaluated experimental trend in Fig. 2 with the generalized seniority calculated trend. The calculations reproduce the experimental trend quite well and resolve this long-standing issue in a simple manner.

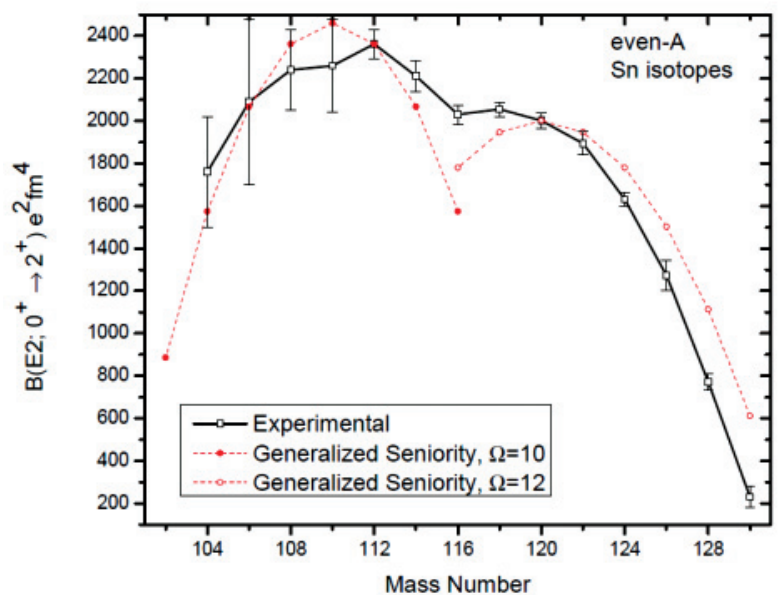

Figure 2: Experimental and Generalized seniority calculated B(E2) trends for the first excited $2^{+}$states, before $\Omega=10$ and after $\Omega=12$ the middle, using the seniority changing transitions in even-even $\mathrm{Sn}$ isotopes.

For calculations, we consider the active valence space of having five neutron orbitals into two parts: (a) $\tilde{j}=g_{7 / 2} \otimes d_{5 / 2} \otimes d_{3 / 2} \otimes s_{1 / 2}, \Omega=10$ and (b) $\tilde{j}=d_{5 / 2} \otimes d_{3 / 2}$ $\otimes s_{1 / 2} \otimes h_{11 / 2}, \Omega=12$. The core has been taken at ${ }^{100} \mathrm{Sn}$ for $\Omega=10$ naturally. We take ${ }^{108} \mathrm{Sn}$ as a core for $\Omega=12$ by assuming completely filled $\mathrm{g}_{7 / 2}$ orbital for the neutron-rich $\mathrm{Sn}$ isotopes. We have used the fact that the $\mathrm{h}_{11 / 2}$ orbital mainly dominates after the middle $\left({ }^{116} \mathrm{Sn}\right)$, while the $\mathrm{g}_{7 / 2}$ orbital completely freezes on reaching ${ }^{116} \mathrm{Sn}$. The ground $0^{+}$states in even-even isotopes is usually attributed as the seniority $v=0$ states. We have calculated these $\mathrm{B}(\mathrm{E} 2)$ values as $\Delta v=2$ transitions from the assumed two parts of active valence space. These calculations result in two asymmetricinverted B(E2) parabolas (See Fig. 2) in terms of different configuration mixings, before and after the middle, which is in tune with the earlier results [25]. The two inverted parabolas corresponding to $\Omega=10$ and 12 , respectively, cross each other and result a dip at ${ }^{116} \mathrm{Sn}$. This is due to the change in the filling of the orbitals resulting in different configuration mixings, before and after the middle. The dip actually highlights the location, where $\mathrm{g}_{7 / 2}$ is now freeze out and $h_{11 / 2}$ starts dominating, not any reduced collectivity etc. [29-31]. These calculations simply resolve this long-standing issue. The goodness of generalized seniority explains the particle number independent energy variation throughout the full chain for these states.

\subsection{An inverted B(E3) parabola for the first excited $3^{-}$states}

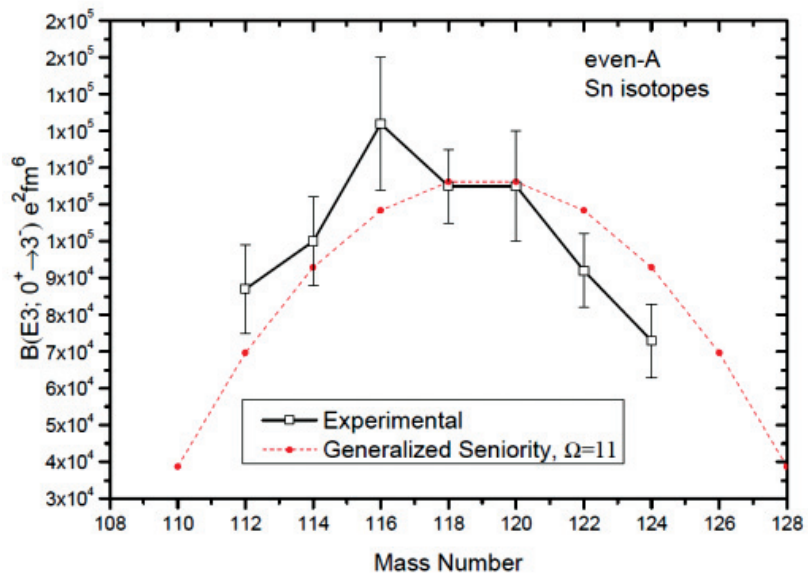

Figure 3: Experimental and Generalized seniority calculated B(E3) trends for the first excited 3- states by using the seniority changing transitions in even-even $\mathrm{Sn}$ isotopes.

The very first excited $3-$ states are usually explained as octupole vibrational states in even-even $S n$ isotopes $[46,47]$. A peak in the middle can be seen in the measured $\mathrm{B}(\mathrm{E} 3)$ values of these states [48]. No theory was able to fully explain this B(E3) trend [27], which motivated us to study these 3 states in view of the generalized seniority scheme. The calculations for these $\mathrm{B}(\mathrm{E} 3) \uparrow$ 
values have been done (as generalized seniority $v=2$ states) by using $\Omega=11$, corresponding to the $d_{5 / 2} \otimes d_{3 / 2} \otimes h_{11 / 2}$. We have calculated the full $\mathrm{B}(\mathrm{E} 3)$ trend by fitting the measured value at ${ }^{118} \mathrm{~S}$. The core has been taken at ${ }^{106} \mathrm{~S} \mathrm{n}$ as $n=0$ situation in these calculations. The calculated results reproduce the known experimental data, see Fig. 3. Experimental data are taken from Kibedi and Spear [48]. A peak in the middle of the $\mathrm{B}(\mathrm{E} 3)$ trend can easily be understood between the seniority changing states. The $d-h$ orbitals having a $\Delta l=3$ connection also sustains the octupole character [19]. However, new measurements are needed, since the present dataset is limited in nature along with large error bars. The generalized seniority scheme strongly supports an inverted parabola for the $\mathrm{B}(\mathrm{E} 3)$ values and expects the unknown values to fall in this order.

\section{Summary and Conclusion}

To conclude, we present a review of our generalized seniority calculations and results in even-even $\mathrm{Sn}$ isotopes for various high spin isomeric states and other excited states. The E1 decaying seniority isomers have been established for the first time. We have explained the similar parabolic behavior of $\mathrm{B}(\mathrm{E} 1)$ and $\mathrm{B}(\mathrm{E} 2)$ values as the goodness of generalized seniority for the $13^{-}$and $15^{-}$ isomers. The scheme strongly guides the configuration mixing behind their generation. The $10^{+}$isomers are now explained as generalized seniority $v=2$ isomers in contrast to the general interpretation in terms of pure seniority. The configuration mixing is essential to obtain the full experimental B(E2) trend of these isomers. The generalized seniority scheme may be used to estimate and predict new isomers.

We have then understood the dip in the middle of the $\mathrm{B}\left(\mathrm{E} 2 \uparrow ; 0^{+} \rightarrow 2_{1}{ }^{+}\right)$trend as different rates of filling the orbitals, before and after the middle, in generalized seniority scheme. However, the generalized seniority remains as a constant at $v=2$ throughout the long chain for the first excited $2^{+}$states. This is how it explains the long standing issue of a double hump in the $\mathrm{B}(\mathrm{E} 2)$ values of these states. We have then explained the $\mathrm{B}\left(\mathrm{E} 3 \uparrow ; 0^{+} \rightarrow 3_{1}^{-}\right)$ trend by using the $d$ and $h$ orbitals in generalized seniority scheme, which also support the octupole vibrational nature of these states.

The generalized seniority results are found to be valid till high-seniority $v=4$ states in even-even $\mathrm{Sn}$ isotopes and are expected to be valid for higher seniority $v=6, \ldots$ states. It can be concluded that the goodness of generalized seniority governs the electromagnetic properties of various states in semi-magic nuclei. Many predictions can be made on this basis. It is particularly important in the long chain of $S n$ isotopes, where performing full scale shell model calculations are rather difficult.

\section{Acknowledgements}

This work has been carried out in IIT Roorkee during BM's Ph.D. thesis under the guidance of Prof. A. K. Jain. Financial support is gratefully acknowledged from Ministry of Human Resource Development (Govt. of India) during her Ph.D. program.

\section{References}

[1] G. Racah, Physical Review 63, 367 (1943). https://doi.org/10.1103/PhysRev.63.367

[2] G. Racah, L. Farkas Memorial Volume 294 (1952).

[3] B. H. Flowers, Proceedings of the Royal Society (London) A, 212, 248 (1952). https://doi.org/10.1098/rspa.1952.0079.

[4] A. K. Kerman, Annals of Physics (NY) 12, 300 (1961). https://doi.org/10.1016/0003-4916(61)90008-2.

[5] K. Helmers, Nuclear Physics. 23, 594 (1961). https://doi.org/10.1016/0029-5582(61)90285-1.

[6] I. Talmi (1993), Simple Models of Complex Nuclei, Harwood Academic.

[7] R. F. Casten (1990), Oxford University Press.

[8] D. J. Rowe, and J. L. Wood (2010), World Scientific Publishing, Singapore. https://doi.org/10.1142/6209.

[9] I. Talmi, Advances in Nuclear Physics 27, 1 (2003), and the references therein.

https://doi.org/10.1007/0-306-47916-8_1.

[10] A. Arima and M. Ichimura, Progress of Theoretical Physics 36, 296 (1966). https://doi.org/10.1143/PTP.36.296.

[11] I. Talmi, Nuclear Physics A 172, 1 (1971). https://doi.org/10.1016/0375-9474(71)90112-6.

[12] S. Shlomo, and I. Talmi, Nuclear Physics A 198, 82 (1972). https://doi.org/10.1016/0375-9474(72)90773-7.

[13] R. Arvieu, and S. A. Moszokowski, Physical Review 145, 830 (1966). https://doi.org/10.1103/PhysRev.145.830.

[14] I. M. Green, and S. A. Moszokowski, Physical Review 139, B790 (1965). https://doi.org/ 10.1103/PhysRev.139.B790.

[15] B. Maheshwari,and A. K. Jain, Physics Letters B 753, 122 (2016) https://doi.org/10.1016/j.physletb.2015.11.079.

[16] B. Maheshwari, A. K. Jain, and B. Singh, Nuclear Physics A 952, 62 (2016). https://doi.org/10.1016/j.nuclphysa.2016.04.021. 
[17] A. K. Jain and B. Maheshwari, Nuclear Physics Review 34, 73 (2017). https://doi.org/10.11804/NuclPhysRev.34.01.073.

[18] A. K. Jain and B. Maheshwari, Physica Scripta 92, 074004 (2017). https://doi.org/10.1088/1402-4896/aa7353.

[19] B. Maheshwari, S. Garg, and A. K. Jain, PramanaJournal of Physics (Rapid Comm.) 89, 75 (2017).

[20] R. Broda et al., Physical Review Letters 68, 1671 (1992) and references therein. https://doi.org/10.1103/PhysRevLett.68.1671

[21] C. T. Zhang et al., Physical Review C 62, 057305 (2000) and references therein.

[22] A. Astier et al ., Physical Review C 85, 054316 (2012). https://doi.org/10.1103/PhysRevC.62.057305.

[23] L. W. Iskra et al ., Physical Review C 89, 044324 (2014). https://doi.org/10.1103/PhysRevC.89.044324.

[24] Evaluated Nuclear Structure Data File: http://www.nndc.bnl.gov/ensdf/

[25] I. O. Morales, P. Van Isacker, and I. Talmi, Physical Review B 703, 606 (2011). https://doi.org/10.1016/j.physletb.2011.08.033.

[26] A. Ansari, Physical Review B 623, 37 (2005). https://doi.org/10.1016/j.physletb.2005.07.031

[27] A. Ansari, and P. Ring, Physical Review C 74, 054313 (2006). https://doi.org/10.1103/PhysRevC.74.054313.

[28] T. Back et al., Physical Review C 87, 031306(R) (2013). https://doi.org/10.1103/PhysRevC.87.031306.

[29] N. Lo Iudice, Ch. Stoyanov, and D. Tarpanov, Physical Review C 84, 044314 (2011).

[30] H. Jiang, Y. Lei, G. E. Fu, Y. M. Zhao, and A. Arima, Physical Review C 86, 054304 (2012). https://doi.org/10.1103/PhysRevC.86.054304.

[31] A. Jungclaus et al., Physical Review B 695, 110 (2011). https://doi.org/10.1016/j.physletb.2010.11.012.

[32] A. Ekstrom et al., Physical Review Letters 101, 012502 (2008). https://doi.org/10.1103/PhysRevLett.101.012502.

[33] P. Doornenbal et al., Physical Review C 90, 061302(R) (2014). https://doi.org/10.1103/PhysRevC.90.061302.
[34] D. C. Radford et al., Nuclear Physics A 746, 83 (2004). https://doi.org/10.1016/j.nuclphysa.2004.09.056.

[35] J. M. Allmond et al., Physical Review C 84,061303(R) (2011). https://doi.org/10.1103/PhysRevC.84.061303.

[36] A. Banu et al., Physical Review C 72, 061305(R) (2005). https://doi.org/10.1103/PhysRevC.72.061305.

[37] J. Cederkall et al., Physical Review Letters 98, 172501 (2007). https://doi.org/10.1103/PhysRevLett.98.172501.

[38] C. Vaman et al., Physical Review Letters 99, 162501 (2007).

[39] P. Doornenbal et al., Physical Review C 78,031303(R) (2008). https://doi.org/10.1103/PhysRevC.78.031303.

[40] R. Kumar et al., Physical Review C, 81, 024306 (2010). https://doi.org/10.1103/PhysRevC.81.024306.

[41] G. Guastalla et al., Physical Review Letters, 110, 172501 (2013). https://doi.org/10.1103/PhysRevLett.110.172501.

[42] V. M. Bader et al., Physical Review C, 88, 051301(R) (2013). https://doi.org/10.1103/PhysRevC.88.051301.

[43] J. M. Allmond et al., Physical Review C, 92, 041303(R) (2015). https://doi.org/10.1103/PhysRevC.92.041303

[44] N. Orce et al., Physical Review C, 76, 021302(R) (2007). https://doi.org/10.1103/PhysRevC.76.021302.

[45] B. Pritychenko, M. Birch, B. Singh, and M. Horoi, Atomic Data and Nucl. Data Tables, 107, 1 (2016). https://doi.org/10.1016/j.adt.2015.10.001

[46] W. Nazarewicz et al., Nuclear Physics A, 429,269(1984). https://doi.org/10.1016/0375-9474(84)90208-2.

[47] P. D. Cottle, Physical Review C, 42, 1264 (1990). https://doi.org/10.1103/PhysRevC.42.1264

[48] T. Kibedi, and R. H. Spear, Atomic Data and Nucl. Data Tables, 80, 35 (2002). https://doi.org/10.1006/adnd.2001.0871. 


\section{Journal of Nuclear Physics, Material Sciences, Radiation and} Applications

Chitkara University, Saraswati Kendra, SCO 160-161, Sector 9-C, Chandigarh, 160009, India

Volume 6, Issue 2

February 2019

ISSN 2321-8649

Copyright: [C 2019 Bhoomika Maheshwari] This is an Open Access article published in Journal of Nuclear Physics, Material Sciences, Radiation and Applications (J. Nucl. Phy. Mat. Sci. Rad. A.) by Chitkara University Publications. It is published with a Creative Commons Attribution- CC-BY 4.0 International License. This license permits unrestricted use, distribution, and reproduction in any medium, provided the original author and source are credited. 